\title{
LA RECEPCIÓN DE ARISTÓTELES Y LA “CUESTIÓN BONAVENTURIANA": DISTINTAS INTERPRETACIONES
}

\author{
ARISTOTLE'S RECEPTION AND THE "BONAVENTURIAN \\ QUESTION”: DIFFERENT INTERPRETATIONS
}

\author{
FRANCISCO JAVIER RUBIO HÍPOLA \\ Universidad Francisco de Vitoria
}

\section{RESUMEN}

La cuestión bonaventuriana aún sigue abierta después de un siglo de debate: ¿es el Seráfico filósofo y teólogo o es solamente un teólogo? ¿Se puede hablar de "filosofía" como ciencia en el pensamiento de san Buenaventura? Íntimamente vinculada a esta cuestión se presenta el problema de la recepción aristotélica. En este artículo se busca organizar las ideas esenciales en torno a esta cuestión y presentar críticamente tres grandes respuestas actuales, para apuntar a cinco conclusiones relevantes. Para lo cual se analizarán las propuestas de E. Gilson, Van Steenberghen, J. Ratzinger y H. U. Von Balthasar desde la generación actual de pensadores medievales: A. Speer, C. Pandolfi y M. Lázaro.

Palabras clave: San Buenaventura, Filosofía, Teología, E. Gilson, J. Ratzinger, Van Steenberghen, H.U. Von Balthasar. 


\section{ABSTRACT}

The Bonaventure's problem is still open after a century of debate: is the Seraphic a philosopher and a theologian or is he just a theologian? Can one speak of "philosophy" as a science in Saint Bonaventure's work? Intimately linked to this question, the problem of Aristotelian reception arises. This article seeks to organize the essential ideas around this issue and critically present three major current answers, to end with five relevant conclusions. For which the proposals of E. Gilson, Van Steenberghen, J. Ratzinger and H. U. Von Balthasar will be analyzed from the perspective of the current generation of medieval thinkers: A. Speer, C. Pandolfi and M. Lázaro.

Keywords: Saint Bonaventure, Philosophy, Theology, E. Gilson, J. Ratzinger, Van Steenberghen, H.U. Von Balthasar.

\section{INTRODUCCIÓN Y METODOLOGÍA}

Las afirmaciones del tipo "Buenaventura es aristotélico" o "Buenaventura es anti-aristotélico" carecen de sentido en el ámbito científico por la generalización y la necesaria imprecisión que implican. No hay dos seres humanos que piensen igual, mucho menos dos grandes pensadores separados por más de un milenio, en dos épocas y en dos lugares tan distintos. En sentido estricto, la identificación de un autor con otro en estas circunstancias es, cuando menos, impropia de una perspectiva académica de la cuestión. Precisamente, uno de los aspectos de más complejo análisis en la historia del pensamiento es la cuestión del binomio heredad-originalidad: en qué medida la obra de un autor queda configurada por una tradición que lo precede y en qué medida es fruto del propio genio. Gran parte de la dificultad de este tipo de comparaciones nace de una perspectiva académica moderna que se enfoca demasiado en el pensador como individuo, y por lo tanto pierde el enfoque más amplio. Sin embargo, así como hay épocas en las que los genios individuales parecen destacar sobre el resto como fulgores resplandecientes en un campo oscuro, también existen épocas en las que el pensamiento se ha dado de una forma mucho más colegiada. Uno de los períodos de la historia en que el desarrollo del pensamiento se ha dado de forma más colegiada o comunitaria es la época del esplendor de la escolástica, entre los siglos XII y XIV, momento en que la "Universitas" adquiere una importancia capital como "encargada de producir saber" ". Estas "corporaciones de profesores y alumnos" daban a la asociación un papel protagónico en la configuración de la enseñanza, el aprendizaje y la producción del saber: "Esta

1 Manuel Lázaro Pulido et al., Historia de la filosofia medieval y renacentista I (Madrid: Editorial UNED-Sindéresis, 2018), 397. 
"asociación" es una característica de la universidad, se trata de un movimiento de comunidad (communitas scholarium, communio magistrorum o societas magistrorum) de colectividad de enseñanzas y agentes educativos"2.

Sin embargo, esta "communitas" no solo se encuentra en la estructura de la universidad como organismo de enseñanza y de desarrollo del pensamiento, sino también en el contrapunto de los fenómenos de la "traditio" y de la "translatio studiorum", por cuanto ésta supone un doble ejercicio de recepción y encuentro de culturas 3 . Por la "traditio" puede descubrirse en toda la Edad Media una línea de continuidad que parte desde la síntesis agustiniana de patrología y neoplatonismo y que articula todo el esfuerzo intelectual escolástico, simbólicamente descrito en el lema -también agustiniano- de "Intellige ut credas; crede ut

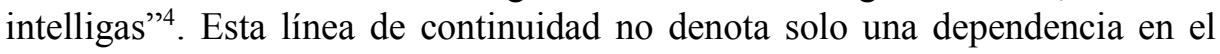
pensamiento de los predecesores, sino ante todo una herencia: los intelectuales medievales se sienten en el camino de la verdad "quasi nanos, gigantium humeris incidentes, ut possimus plura eis et remotiora videre, non utique proprii visus acumine, aut eminentia corporis, sed quia in altum subvenimur et extollimur magnitudine gigantea" 5 . En cambio, por la "translatio studiorum" el pensador escolástico occidental (especialmente a partir del siglo XII y hasta finales del siglo XIV) descubre el bagaje filosófico de una "traditio" alternativa que había conservado obras importantes de Aristóteles perdidas en occidente y que se había ido construyendo en diálogo con ellas. Esta "traditio" alternativa que parte del pensamiento bizantino, suma el desarrollo intelectual de los árabes y la mediación de la filosofía judía llega al occidente cristiano por distintos factores históricos a mediados del siglo XII. El pensamiento medieval se vio muy beneficiado por los problemas y el legado de esta "tradición alternativa": fue necesario dialogar con ella, incorporarla a nuevas síntesis, aceptar y matizar un nuevo instrumental metafísico y esto generó nuevas vías de desarrollo intelectual.

A estas dos formas de "communitas" es preciso añadir una tercera en el caso concreto de san Buenaventura: la que conforman quienes siguen la regla de una orden. En efecto, otro de los fenómenos característicos de esta etapa final de la Edad Media es la aparición de las órdenes mendicantes, una nueva forma de consagración que responde a las nuevas necesidades pastorales surgidas por

2 Lázaro Pulido et al., Historia de la filosofia medieval y renacentista I, 397.

3 Una explicación completa de este fenómeno puede encontrarse en: Alain de Libera, $L a$ philosophie médiévale (Paris: Presses universitaires de France, 1993); Francisco León Florido, Las filosofías en la Edad Media: crisis, controversias y condenas (Madrid: Biblioteca Nueva, 2010), 90-92.

4 Agustín de Hipona, Sermones, XLIII, c.7, n.9 (PL 38, 200).

5 Juan de Salisbury, Metalogicum, III, c.4-C (PL 199, 200). (Cita atribuida a Bernardo de Chartres). 
las circunstancias históricas de la época, poniendo especial acento en el respeto a la ortodoxia y en el servicio a la Iglesia ${ }^{6}$. Las dos principales órdenes serán la de los dominicos y la de los franciscanos. Parece preciso subrayar la característica común carismática que define cada una de estas órdenes: en el caso de los primeros, "el dominico se entrega a la ascesis en la vida comunitaria para estar mejor preparado a la acción y al servicio, centrado en la oración, la predicación, la dirección espiritual y la enseñanza"” . La orientación de toda esta actividad es marcadamente contra la herejía. En el caso de los franciscanos también busca la predicación, pero orientada hacia la conversión del corazón a una vida de penitencia, de amor y de pobreza. La orden franciscana no estuvo tan volcada al estudio como la dominicana, pero dio a la historia importantes intelectuales. Para comprender la "forma mentis" de un pensador perteneciente a una de estas órdenes es preciso comprender la importancia del vínculo carismático que lo une al espíritu de la misma.

Esta triple "communitas" - en la estructura escolar de la "universitas", en las líneas convergentes de la "traditio" y la "traslatio", y en la comunión carismática- sitúa el "quaesitum" del presente trabajo en un marco hermenéutico muy complejo: la relación entre dos pensadores situados en contextos culturales tan distintos y con métodos y objetivos intelectuales tan diversos es, de por sí, una tarea azarosa, por más explícita que sea dicha relación. Esta relación resulta tan particularmente problemática, que ha recibido su propia etiqueta en la tradición de estudio medieval: "la cuestión bonaventuriana". A todo esto es preciso sumar la evolución de cada uno de los pensadores (la repetida taxonomía de obras de juventud y obras de madurez) y cómo dicha evolución ha interferido en la relación. Por supuesto que, además, Buenaventura nunca escribió ni concibió una obra monográfica sobre algún aspecto del pensamiento del Estagirita, por lo que el uso que hace de su pensamiento es siempre funcional a otro problema. Eso requiere analizar pormenorizadamente el contexto del original en referencia al contexto en el que el Seráfico incluye la cita en cuestión. Por ejemplo, cuando en el Comentario al III libro de las Sentencias, Buenaventura incluye una referencia a la Metafisica de Aristóteles ${ }^{8}$ para definir la "sapientia" como una ciencia de las causas sublimes. En este caso el Seráfico abusa ligeramente del contexto real de la cita para poder emplearlo en beneficio del argumento que se trae entre manos. Este "abuso" por supuesto, deja de ser tal si consideramos lo diferente que resulta, por los distintos

6 Cf. Lázaro Pulido et al., Historia de la filosofia medieval y renacentista I, 398-402.

7 Lázaro Pulido et al., Historia de la filosofía medieval y renacentista I, 400.

8 Cf. Nota n.3, de Buenaventura, III Sententiarum, d. 35, q.1, a.1 (ed. Quaracchi, III, 774). La cita es de Aristóteles, Metafisica, I, c.1 (981b, 27-28). 
contextos gnoseológicos y epistemológicos, la noción de "filosofia" sobre la que trabajó Aristóteles, Buenaventura y, sobre todo, la noción de "filosofía" a la que recurrimos desde la perspectiva contemporánea para establecer un análisis. Acaso la pregunta de fondo a todo el problema sea si existe una "filosofía bonaventuriana" y si esta es compatible o siquiera comparable con una "filosofía aristotélica". Todo esto, por supuesto, sin contar con las vicisitudes históricas. La recepción de Aristóteles por Buenaventura no se dio en un momento precisamente tranquilo en el mundo de la universidad occidental. Concretamente en la Universidad de París las tensiones entre la corriente de averroístas latinos (especialmente vinculada a la facultad de artes) ${ }^{10}$ y la mayoría de los profesores de la facultad de teología dio lugar a una serie de condenas a tesis aristotelizantes consideradas contrarias al dogma cristiano por parte de la Iglesia ${ }^{11}$. Si a esto añadimos la clara prominencia que tienen san Francisco (por su "communitas" franciscana) y san Agustín (por su "communitas" con la "traditio") en el pensamiento de san Buenaventura, y el fenómeno -ya de suyo complejo- de la recepción del aristotelismo en occidente, parece que el problema solo se acrecienta y no puede resolverse sino en un estudio mucho más amplio que pueda aportar en sus conclusiones toda la fertilidad de pensamiento que caracteriza la síntesis teológica llevada a cabo por el Seráfico.

Por fortuna se han realizado trabajos de estas características. Hay estudios que son ya clásicos en este tema (como el análisis de J. Ratzinger ${ }^{12}$, el de H.U. Von Balthasar $^{13}$, la historia de É. Gilson ${ }^{14}$ o la de F. Van Steenberghen ${ }^{15}$ ), dichos estudios han sido convenientemente revisados y nos encontramos en la

9 Cf. Andreas Speer, "Bonaventure and the Question of a Medieval Philosophy", Medieval Philosophy \& Theology 6 (1997): 25-29.

10 Gilson analiza brevemente y con precisión las motivaciones que diferenciaban a los maestros de ambas facultades: cf. Étienne Gilson, La filosofia en la Edad Media (Madrid: Editorial Gredos, 2014), 368-69. Estudios más recientes tienden a restar valor a estas diferencias o, al menos, a cuanto en ellas supone una división entre una cierta concepción "autónoma" de la filosofía y la teología: cf. Jan A. Aertsen, "Gibt Es Eine Mittelalterliche Philosophie", Philosophisches Jahrbuch 102 (1995): 161176.

11 Para analizar con detalle el papel que jugó san Buenaventura en medio de este clima se recomiendan dos referencias científicas actualizadas: Florido, Las filosofias en la Edad Media: crisis, controversias y condenas, 186-91. Manuel Lázaro Pulido, "La lectura bonaventuriana de Aristóteles desde san Francisco", Pensamiento: Revista de investigación e Información filosófica 275 (2017): 103114.

12 Joseph Ratzinger, Obras completas de Joseph Ratzinger, II: Comprensión de la revelación y teología de la historia de San Buenaventura, vol. II (Madrid: Biblioteca de Autores Cristianos, 2013).

13 Hans Urs von Balthasar, Gloria. Una estética teológica, Estilos eclesiásticos. Ireneo, Agustín, Dionisio, Anselmo, Buenaventura, vol. II (Madrid: Encuentro, 1992).

14 Etienne Gilson, La Filosofia de San Buenaventura (Buenos Aires: Desclée de Brouwer, 1948).

15 Fernand Van Steenberghen, Epistémologie (Louvain: Ed. de l'Inst. Supérieur de Philosophie, 1956); Fernand van Steenberghen, Aristotle in the West: the origins of latin Aristotelianism = Aristote en Occident. English (Louvain: Nauwelaerts Publishing House, 1970). 
actualidad con un panorama de gran interés: un conjunto de filósofos de segunda generación que se encuentran revitalizando los estudios bonaventurianos y actualizando su propuesta en diálogo con el pensamiento de hoy. Entre ellos he escogido a tres autores destacados que pertenecen a círculos e idiomas diversos, dentro de lo que cabe, y que han afrontado explícitamente la cuestión de la recepción de Aristóteles por san Buenaventura. La selección de estos autores ha seguido principalmente el criterio de especialización, especialmente por la calidad de las publicaciones sobre el tema y la posibilidad de poner sus conclusiones en diálogo. En el presente trabajo se buscará realizar un análisis del estudio de los doctores y académicos especialistas en el tema A. Speer, C. Pandolfi y M. Lázaro Pulido y se concluirá con una crítica de conjunto y con un intento de solución "reductiva" del problema planteado.

\section{RESULTADOS}

1. "Bonaventure And the Question of A Medieval Philosophy"16, ANDREAS SPEER

Ante el doble interrogante con el que Speer cierra la conclusión de su artículo ("does Bonaventure have his own unique approach to philosophy? In fact, does he have a philosophy at all?" $\left.{ }^{17}\right)$, la tesis del filósofo alemán consiste en que el Seráfico tiene una propuesta filosófica que consiste en una forma de conocimiento provisional de la verdad - como también lo es la teología- que debe reducirse en primer lugar al saber teológico y en segundo lugar a la contemplación de Dios ${ }^{18}$. Esta "reductio" o referencialidad, no debe entenderse como un mero servicio como "ancilla theologiae", sino que el mismo Buenaventura reconoce la necesidad de que la teología (al menos la capacidad del conocimiento humano para conocer la verdad teológica) sea analizada por la filosofía. Para formular esta tesis Speer no recurre a los primeros escritos de Buenaventura, que suelen etiquetarse como los más universitarios o académicos, sino que basa su análisis en las Collationes in Hexaëmeron y en las Collationes de Donis, escritos de madurez. Esta filosofía ocupa en algunos textos bonaventurianos el rango más alto de "ciencia especulativa" ("(..) scientia philosophica nihil aliud est quam veritatis ut scrutabilis notitia certa" ${ }^{19}$ ) dejando a la teología el rango de "ciencia de la sagrada Escritura" ("(...) scientiam theologicam, quae est veritatis credibilis notitia pia (...). Ideo super fidem fundata est

16 Speer, "Bonaventure and the Question of a Medieval Philosophy".

17 Speer, "Bonaventure and the Question of a Medieval Philosophy", 29.

18 Cf. Speer, "Bonaventure and the Question of a Medieval Philosophy", 44.

19 Buenaventura, Collationes de septem donis Spiritus, col. IV, n.5 (ed. Quaracchi, V, 474). 
scientia theologica: sicut scientiae philosophicae super prima principia fundatur, ita scientia Scripturae fundatur super articulos fidei, $\left.(\ldots)^{n 20}\right)$.

Bajo esta perspectiva la problemática derivada de la hermenéutica en torno a la recepción del pensamiento del Estagirita por san Buenaventura es relativa a la función que desempeña dicho pensamiento en el argumento teológico en cuestión. Parece claro que el gran problema filosófico en este sentido es de carácter gnoseológico, aunque el mismo Speer subraya otros importantes temas que con frecuencia se descuidan: si la búsqueda de un fundamento veritativo de la teología es una cuestión de inspiración principalmente anselmiana, su objetivo especulativo es de inspiración victorina (en especial de Hugo de San Víctor) y su énfasis histórico-salvífico es herencia de Joaquín de Fiore ${ }^{21}$. En todo caso, detrás de estos tres temas aparece siempre el telón de fondo del agustinismo medieval.

Para Speer los historiadores intelectuales (como De Libera ${ }^{22}, \operatorname{Imbach}^{23} \mathrm{o}$ Flasch ${ }^{24}$ ) yerran al acentuar una división rígida entre filosofía y teología, más si cabe- al tratar de presentar en clave histórica el esfuerzo intelectual medieval en una progresiva búsqueda de esta autonomía respecto a la teología o viceversa. La autonomía de la filosofía -alcanzada en la actualidad-, su independencia como saber autónomo deslindado de cualquier otro saber, centrada en la cuestión de la existencia propia y de la realidad, parece convertirse en un complicado e infértil juego intelectual cuyas reglas y logros pertenecen a una comunidad cada vez más reducida y especializada ${ }^{25}$. Cabe discutir incluso si la filosofía aristotélica no solo tiene ella misma una funcionalidad hacia la ética, la física o la polis (es decir, está lejos de poder considerarse autónoma), sino también teológica ${ }^{26}$.

Tampoco Gilson acierta en su lectura de la recepción de Aristóteles por parte de san Buenaventura. Speer valora la intuición gilsoniana de comprender la obra del Seráfico como quizá la mayor síntesis de pensamiento cristiano jamás llevada a cabo y como quizá la más peculiarmente medieval. Para el filósofo parisino parece claro que la comprensión puramente racionalista-funcional de la

20 Buenaventura, Collationes de septem donis Spiritus, col. IV, n.13 (ed. Quaracchi, V, 476).

21 Cf. Speer, "Bonaventure and the Question of a Medieval Philosophy", 43.

22 Alain de Libera, Penser au Moyen Age (Paris: Editions du Seuil, 1991).

23 Ruedi Imbach, Gli Studi Di Filosofia Medievale Fra Otto E Novecento (Roma: Ed. di Storia e Letteratura, 1991).

24 Kurt Flasch y Etienne Tempier, Aufklärung im Mittelalter?: Die Verurteilung von 1277 : das Dokument des Bischofs von Paris (Mainz: Dieterich, 1989).

25 Cf. Speer, "Bonaventure and the Question of a Medieval Philosophy", 43-44.

26 Cf. Pierre Aubenque, "Aristóteles y el problema de la metafísica", Logos. Anales del Seminario de Metafisica 50 (24 de julio de 2017): 9-19. 
filosofía que se tiene en la modernidad no debe ser el criterio para juzgar si un pensador medieval era o no filósofo. De hecho, Buenaventura no puede llamarse "filósofo" desde una perspectiva racionalista porque el Seráfico rechaza el Organon aristotélico como criterio último de verdad en las cuestiones filosóficas. Este es para Gilson el principal elemento que diferencia el pensamiento del Seráfico del pensamiento del Angélico ${ }^{27}$. Para Speer este criterio es reducido y obvia la misma comprensión del autor sobre lo que la filosofía es en el contexto original de su propia síntesis. El esfuerzo por separar la "forma mentis" bonaventuriana de la tomista - para salvaguardar la originalidad de ambos en sus respectivas síntesis- provoca que Gilson pierda de vista otras posibilidades epistemológicas para la filosofía perfectamente válidas (al menos como planteamiento inicial) más allá de un punto de partida meramente racional o lógico (sin influencia de la teología). Dicho de otro modo, no se contempla la posibilidad de que la filosofía pueda iniciar su andadura con pleno derecho dentro de un contexto en el que el principio ontológico sea: "esse enim divinum primum est, quod venit in mente"28; y el principio gnoseológico sea: "nihil sciatur nisi per veritatem immutabilem" 29 .

Van Steenberghen se levantó contra este intento de "reducir" el pensamiento de Buenaventura a una metafísica de la mística cristiana y franciscana. El historiador y filósofo belga en su célebre historia del pensamiento en el siglo XIII, según Speer, enmarca el pensamiento de Buenaventura según tres grandes características: el Seráfico es un profesor universitario y, como tal, depositario de formación escolástica y en gran medida aristotélica; su acercamiento a la filosofía es claro, pero ecléctico en sus raíces; y sus principales contribuciones intelectuales son de naturaleza teológica. Speer parece estar de acuerdo con Van Steenberghen a la hora de restar importancia a la influencia de lo que llama: "some fuzzy inferences from a still fuzzier notion of "Franciscan spirituality"“30. Además, acepta igualmente que las raíces filosóficas del pensamiento del Seráfico emanan de problemas principalmente teológicos y que son de naturaleza ecléctica: responden a un cierto modus aristotélico -especialmente en su presentación formal-, pero se nutren de las fuentes clásicas de la escolástica medieval. Sin embargo, Speer relativiza en gran medida la importancia de la influencia total del Estagirita en el pensamiento de Buenaventura, a favor de la

27 Cf. Speer, "Bonaventure and the Question of a Medieval Philosophy", 26-27.

28 Buenaventura, Collationes in Hexaëmeron, col. X, n.6 (ed. Quaracchi, V, 378).

29 Buenaventura, Collationes in Hexaëmeron, col. I, n.13 (ed. Quaracchi, V, 331).

30 Speer, "Bonaventure and the Question of a Medieval Philosophy", 28. Speer enfatiza el problema. Para él la consideración de la "vocación franciscana" ha empañado el estudio del pensamiento del Seráfico. Mucho más fértil habría resultado -afirma poco después- el estudio sobre la relación entre Buenaventura y Alejandro de Hales, por ejemplo. 
prominencia de Agustín, Alejandro de Hales, Anselmo o incluso de los Victorinos.

La última voz analizada por Speer es la de J. Ratzinger -papa Benedicto XVI- especialmente en el ya clásico texto de habilitación de doctorado. Ratzinger hace algo muy similar: dedica no pocas páginas y apartados a una crítica de las interpretaciones precedentes, de manera especial a las de Gilson y Van Steenberghen. Speer no dedica un apartado como tal al análisis de la visión de Ratzinger, pero sí intercala varios comentarios al respecto a lo largo de su artículo. Speer critica que la interpretación dominante sobre uno de los textos principales de Buenaventura (las Collationes in Hexaëmeron), heredada de Ratzinger, según la cual esta obra manifiesta un anti-aristotelismo, un anti-filosofismo e incluso un anti-escolasticismo que contrasta netamente con el quehacer de la gran mayoría de los contemporáneos del Seráfico, incluyendo al Angélico. Para Speer, por el contrario, pocos textos proporcionan una mirada filosófica más concisa y concreta en la bibliografía bonaventuriana como la primera "collatio". Los cuatro grandes pilares de la consideración filosófica bonaventuriana aparecen en las Collationes: "nec aliquo modo aliqua veritatem potest nisi per illam veritatem"31 (la necesaria existencia de la verdad para que se dé el conocimiento); "nihil sciatur nisi per veritatem immutabilem" 32 (la certeza de tal verdad depende de la inmutabilidad del objeto); "idem est principium essendi et cognoscendi" 33 (se da una proporcionalidad entre la estructura ontológica y la estructura cognoscitiva); "esse enim divinum primum est, quod venit in mente" 34 (el "esse divinum" es el primer concepto de la mente, el "a priori" que posibilita todo otro conocimiento). Yerra por lo tanto Ratzinger al enfatizar, de nuevo, la división entre teología y filosofía en el contexto del pensamiento bonaventuriano. Especialmente al considerar el íter vital del santo de Fidanza como un despegue desde el escolasticismo universitario (académico, escolástico y aristotélico) hacia una etapa de madurez "extra-universitaria" (anti-filosófica y anti-aristotélica).

\section{BonaVEnTURA: "Forme DEL PENSARE CRISTIANO" 35 , CARMELO} PANDOLFI

El profesor y experto medievalista Carmelo Pandolfi, en su monografía titulada "Forme del pensare cristiano" dedica un largo capítulo a los "Cuatro

31 Buenaventura, Collationes in Hexaëmeron, col. I, n.13 (ed. Quaracchi, V, 331).

32 Buenaventura, Collationes in Hexaëmeron, col. I, n.13 (ed. Quaracchi, V, 331).

33 Buenaventura, Collationes in Hexaëmeron, col. I, n.13 (ed. Quaracchi, V, 331).

34 Buenaventura, Collationes in Hexaëmeron, col. X, n. 6 (ed. Quaracchi, V, 378).

35 Carmelo Pandolfi, Forme del pensare cristiano: Agostino, Anselmo, Bonaventura, Tommaso, Richerche di storia della filosofia e teologia medioevali 4 (Roma: If Press, 2016). 
grandes intérpretes del pensamiento de San Buenaventura"36. Estos cuatro son: É. Gilson, F. Van Steenberghen, H.U. Von Balthasar y J. Ratzinger.

Sobre el primero, Pandolfi aclara que la distinción propuesta por Gilson entre el Seráfico y el Angélico no debe entenderse como una distinción entre un racionalista y un fideísta. La distinción es de carácter más descriptivo que esencial porque para el autor ambos pertenecen antes a la "traditio" medieval que a la heredad aristotélica. Pandolfi lo expresa bellamente: "(...) la distinción entre ambos Doctores del s. XIII resulta relevante para la investigación solo por su diversa interpretación del papel de las dos voces en el unísono fe-razón"37. Lejos de restar originalidad, para Gilson, esta diversidad se arraiga en la misma mirada metafísica de ambos y connota, por lo tanto, dos estilos muy diversos de quehacer filosófico y teológico (por ejemplo, entre una mirada más analógicoontológica y una mirada más anagogógica-reductiva). Dicho de otro modo, para Gilson, ante los peligros filosóficos que parecían renacer en los últimos siglos medievales, se alzan dos propuestas sistemáticas y sintéticas alternativas: el tomismo y el agustinismo del siglo XIII - entre cuyas filas destaca el Seráfico doctor-. En cualquier caso, ninguno de los dos hace filosofía o teología autónoma. Además, acierta Gilson al ofrecer el pensamiento de Buenaventura como "una síntesis de filosofía cristiana", es decir: la especulación filosófica se origina en la experiencia de la fe en la verdad revelada ${ }^{38}$. Esta "filosofía cristiana" supone una serie de contenidos temáticos originalmente bonaventurianos: el mundo como "expresión de Dios"; la "notitia Dei" constituida en el ente; y la fuerza gnoseológica de la doctrina de la iluminación ${ }^{39}$. Más que corregir, Pandolfi matiza a Gilson: el hecho de que estas aportaciones puedan presentarse como "filosofía cristiana" por su origen, no tiene por qué no poder considerarse como materia filosófica "per se". Por lo tanto, no es necesario reducir el significado de "filósofo" para poder aplicarlo a Buenaventura. No es imprescindible una mirada originalmente aristotélica -para Pandolfi ese tampoco es el caso con Tomás de Aquino- para que un filósofo medieval pueda ser considerado tal. En definitiva, para Pandolfi, es oportuna la visión ontológica del mundo como expresión de Dios -que conlleva la emergencia del "esse divinum" en los entes y una doctrina de la iluminación- pero para que esa mirada no caiga en el gnosticismo, es preciso también un soporte teológico -no solo como causa final, sino ante todo como causa fundante. Una filosofía del "Logos" requiere una metafísica del Bien y, por lo tanto, una culminación Trinitaria que dé sentido al

36 Pandolfi, Forme del pensare cristiano, 131-225.

37 Pandolfi, Forme del pensare cristiano, 138. Las traducciones del original italiano son del autor del presente artículo.

38 Cf. Gilson, La filosofia en la Edad Media, 531.

39 Cf. Pandolfi, Forme del pensare cristiano, 152. 
Amor definitivo que lo explica y lo recoge todo (a lo que todo se reduce) ${ }^{40}$. Pandolfi, al fin, afirma que Gilson acierta también al proponer como parte importante de la síntesis original bonaventuriana la herencia del espíritu franciscano.

Este es el primer punto criticado por Van Steenberghen a Gilson. Pero Pandolfi analiza primero el punto en el que puede estar de acuerdo con el pensador belga. Al haber propuesto gran parte de su propuesta en clave de polémica respecto a Gilson, resulta más sencillo llevar a cabo un análisis de su postura. En primer lugar, Pandolfi está de acuerdo con la crítica que Van Steenberghen juzga la mirada "exageradamente agustinista" de Gilson sobre Buenaventura. El Seráfico se diferencia del Doctor de Hipona mucho más de lo que Gilson da a entender: lo supera especialmente en la estructura interna sintética de la sabiduría cristiana (que es para Van Steenberghen el problema más delicado del siglo XIII ${ }^{41}$ ). Pero las conclusiones a las que el pensador belga llega por este camino no son del todo aceptables. La cuestión, en el fondo, es si en un pensador medieval puede "diseccionarse" el filósofo del teólogo. Este punto, especialmente en el caso que nos ocupa, no es baladí. Precisamente la perspectiva gilsoniana de presentar a Buenaventura como destacado autor de una "síntesis filosófica cristiana", implica una distinción de base entre dos dimensiones -no confusas, sino armonizables-: la vía racional-filosófica y la vía fiduciaria-teológica. El hecho de que exista la distinción implica necesariamente que existe un "problema bonaventuriano" que se resuelve en la explicación de la colocación y de la armonía entre ambas dimensiones ${ }^{42}$. Es decir, que la "reductio" que se hace al Principio (casi literalmente filo-Sofía) existe por la convergencia -que no se da en grado sumo- de signos plurales, creaturales y cristianos ${ }^{43}$. Si no se da la distinción la "reductio" debe entenderse en una clave unívoca, mucho menos fecunda y no del todo consistente con las palabras mismas del Seráfico.

En otro orden de cosas, Pandolfi concede a Van Steenberghen el acierto a la hora de analizar la relación "genética" entre Aristóteles y Buenaventura. Al haber abandonado de manera temprana la universidad de París, el Seráfico no asistió a

40 Cf. Pandolfi, Forme del pensare cristiano, 156-59. Para el medievalista italiano gran parte de los "defectos" de la interpretación gilsoniana se deben a lo temprano de esta obra en su propio desarrollo intelectual (1924). Años después cuando madure su pensamiento -especialmente sobre la concepción del "esse" tomista y su superación respecto al Filósofo- estas conclusiones limitadas se habrían podido superar.

41 Cf. Fernand Van Steenberghen, La philosophie au XIIIe siècle (Louvain-la-Neuve: Institut Superieur de Philosophie, 1991), 176.

42 Para Pandolfi, Van Steenberghen está autorizado a no aceptar el problema bonventuriano por no aceptar el punto de partida de Gilson: "que la Revelación re-consiente el origen (o consiente un nuevo origen) de la filosofia" (Pandolfi, Forme del pensare cristiano, 174.)

43 Cf. Pandolfi, Forme del pensare cristiano, 174. 
la explosión del pensamiento de Aristóteles en la misma por la novedad de obras como la Metafísica o el De Anima. Sí conoció, seguramente, la Magna Moralia y el Canon Logicus, que perduraban en la tradición medieval occidental ${ }^{44}$. Por lo tanto, Buenaventura ni desprecia ni aprecia especialmente al Filósofo: simplemente no lo ha estudiado tan a fondo. Pandolfi está fundamentalmente de acuerdo con esta línea de pensamiento, que considera contingente respecto al problema de fondo. A pesar de lo cual asistimos en numerosos pasajes bonaventurianos a un genuino elogio de la figura o del pensamiento del Filósofo. Este hecho lo constata el pensador belga e insiste en él tratando de connotar una simpatía que a Pandolfi le parece cuando más accidental: "Estos [los materiales aristotélicos en la obra de Buenaventura] deberían ser coherentemente interpretados como el uso de una lengua aristotélica, que estaba de moda, para fines pastorales y hermenéuticos, y no como una adhesión inicial a la "pars veri" del Aristotelismo" ${ }^{45}$. Las conclusiones de Van Steenberghen en este sentido son de gran interés, aunque sean difícilmente asumibles con los estudios actuales: si Gilson defiende que Buenaventura y Tomás representan dos miradas diversas que se oponen al común enemigo del aristotelismo latino, para el filósofo belga ambos son dos variantes específicas (en la parte filosófica de su obra) que pertenecen a una rama del aristotelismo latino: el aristotelismo neoplatonizante latino ${ }^{46}$. Para Pandolfi ambas propuestas son inexactas (más la de Van Steenberghen que la de Gilson). En cualquier caso, Van Steenberghen defiende que en ambos casos (con el Tomismo y el Bonaventurismo) la filosofía de base que sustenta los sistemas es la aristotélica. Por lo tanto, no solo son visiones filosóficas cercanas (apenas diferenciadas por la "creatio ab aeterno de ratione"/ "de fide" y por el "binarium"), son también comparables. Entre ambas, destaca la propuesta tomasiana por su lucidez. Por lo tanto, parece legítimo estudiar a Buenaventura siempre en referencia a Tomás (siguiendo el estilo inmediatamente posterior a la Aeterni Patris). Según Pandolfi esta propuesta filológico-hermenéutica se encuentra poco sustanciada, principalmente por cuanto omite. Parece cierto que ambas metafísicas proponen una unidad de aristotelismo (sínolo) y de platonismo (participación). Pero esta característica ni define ni agota ninguna de las dos propuestas. Para empezar, obvia todas las diferencias en perspectiva, en fundación epistemológica y en presentación teológica. Y principalmente, obvia la definición misma de "metaphysicus" en el ámbito estricto del pensamiento bonaventuriano, que posee connotaciones propias: "el "verus metaphysicus" es aquel que realiza el "itinerarium ad Deum" por

44 Para una mayor profundización en el destino medieval del pensamiento del Estagirita se recomienda: Florido, Las filosofias en la Edad Media: crisis, controversias y condenas.

45 Pandolfi, Forme del pensare cristiano, 176.

46 Cf. Pandolfi, Forme del pensare cristiano, 176. El autor se apoya en: Steenberghen, La philosophie au XIIIe siècle, 220-21. 
los puntos siempre vinculados de multiformas visuales microcósmicas y macrocósmicas, epistemológicas, históricas, e incluso técnicas y políticas (donde se encuentran los entes y el Evento cristiano)" ${ }^{\prime 47}$.

La gran aportación hermenéutica de Von Balthasar, para Pandolfi, consiste en haber aportado una perspectiva original al estudio del Seráfico. En primer lugar por haber descubierto en él uno de los más grandes tratados sobre el "Pulchrum" en el vínculo entre Trinidad y belleza. El célebre filósofo alemán insiste en el carácter sintético del pensamiento bonaventuriano, más allá de lo afirmado por Gilson o Ratzinger. Para Pandolfi, Von Balthasar descubre en Buenaventura (y su tesis nuclear sobre el Verbo como expresión eterna del Padre y por lo tanto como Ser modélico, Belleza y Drama) la formulación teológicomística de la intuición puramente mística de Adrienne Von Speyr ${ }^{48}$. Para el filósofo de Lucerna el Seráfico logra presentar reductivamente una reflexión sobre la belleza, que puede decirse teológica como síntesis de todas las vías de signos creaturales y cristianos. En este sentido, la metafísica de Buenaventura no puede estudiarse como una suma de fórmulas estructurales vacías de contenido, sino como una proclamación de la belleza de la creación como una efusión de Dios, a la vez revelación y participación trinitaria. Así el Seráfico supera y a la vez implica a san Agustín, Dionisio Areopagita, los Victorinos, Aristóteles, etc., pero siempre bajo el signo culminante de la cruz franciscana (el momento del éxtasis franciscano de Alvernia), símbolo a su vez del Amor que resucita y recoge, que representa el "axis" espacial y temporal del mundo y de la historia (toda la creación). Todo esto sin caer en una forma de presentación "apofática" de Dios": la paradoja "abisal" de la revelación divina en Cristo consiste precisamente en que cuanto más se revela la Trinidad (más se da en amor) más valor adquiere su misterio. Dicho de otro modo: más insondable y vasta resulta la gratuidad de su amor. A partir de esta clave central, resulta más lógico analizar los vínculos del Seráfico con otros grandes pensadores cristianos: con el Angélico (entendiendo su metafísica en clave intensiva) ${ }^{50}$, con la teología histórico-escatológica de Joaquín de Fiore, con las intuiciones filosóficas (gnoseológicas y ontológicas) agustinianas, con la teología trinitaria de los

47 Pandolfi, Forme del pensare cristiano, 178.

48 Cf. Pandolfi, Forme del pensare cristiano, 180.

49 Von Balthasar defiende también a Buenaventura de toda posible acusación de ontologismo o de reducir a Dios a mero principio o causa de la realidad. Al afirmar que el conocimiento de Dios se da también "sicut objectum et ratio motiva", -Buenaventura, Sermones selecti de rebus theologici, sermo IV, n.18 (ed. Quaracchi, V, 572)-, no debe entenderse (como hace Gilson) que Dios es un puro "medio" de conocimiento no objetivado (cf. Von Balthasar, Gloria, II, 295).

50 Para Von Balthasar la radical diferencia entre ambos (a favor del Seráfico) consiste en que Tomás de Aquino resuelve la "noesis" en la dinámica abstractiva del conocimiento, pero le falta la captación del carácter objetivo-declarativo de la verdad. Cf. Von Balthasar, Gloria, II, 270. 
Padres Griegos, y, principalmente con la filosofía y la teología que surgen del san Francisco estigmatizado. En definitiva, dada la clave de lectura con la que Von Balthasar interpreta el pensamiento del Seráfico hay poco espacio para considerar el influjo de Aristóteles en el mismo. Sin embargo Pandolfi lo incluye en la lista, especialmente por dar más peso a la herencia franciscana y por matizar algo de las aportaciones de Gilson y Ratzinger.

Por último, Pandolfi considera la aportación de este último. En primer lugar analiza su "toma de postura" frente al debate Gilson-Van Steenberghen. Al filósofo francés le da la razón al defender la negación bonaventuriana de la división entre filosofía y teología, al defender su independencia respecto al Angélico y al presentar la consideración del pensamiento bonaventuriano en clave fundamentalmente cristocéntrica. Al segundo le da la razón, en cambio, al defender la imposibilidad de defender como "agustiniano" el pensamiento de Buenaventura. De nuevo, en contra del filósofo belga, Ratzinger defiende el "antiaristotelismo" de Buenaventura (tema al que dedica el capítulo IV de su tesis de habilitación). Pandolfi hace un esfuerzo por matizar las etiquetas de anti-aristotelismo y de anti-tomismo que el joven Ratzinger cuelga con tanta facilidad y profusión en los hombros de Buenaventura. Para el medievalista italiano, Ratzinger simplemente constata el hecho histórico de que, en su madurez, Buenaventura insistió tanto en prevenir a los miembros de su orden de las corrientes universitarias de las tentaciones del aristotelismo latino, como en advertir el peligro de tres tesis tomasianas: sobre la simplicidad de los espíritus puros, la diferencia real del alma y facultades, y el célebre problema sobre la hipótesis de la eternidad del mundo. Es decir, para Ratzinger no existe acusación formal por parte de Buenaventura a Tomás de Aquino por una "heterodoxia aristotélica". Más bien hay un aviso y una toma de posición frente a los peligros de una serie de tesis aristotélicas. Sin embargo, para Pandolfi el estudio de Ratzinger no trata simplemente de justificar una toma de postura intermedia entre Gilson y Van Steenberghen, sino que apunta a algo más: la dotación de un sentido históricoteológico pleno al pensamiento bonaventuriano. Es decir: más allá de los límites del debate hermenéutico existe una vía de interpretación del pensamiento del Seráfico que supera dichos límites "por arriba". Para acceder a esa clave de interpretación, Ratzinger concentra la perspectiva de la diferencia entre Tomás de Aquino y Buenaventura, más que en un "Aristóteles: sí o no" o un "Agustín: sí o no", en el ámbito de la teología de la historia (clave anagogógica-reductivaescatológica). En esto consiste la originalidad de la mirada de Ratzinger ${ }^{51}$. 
Para el teólogo alemán esta nueva vía supone la superación tanto de la autonomía del pensamiento en sí (aristotelismo, en esencia) como de cualquier forma de teología meramente racional o científica en sentido reducido. Todo ello, por supuesto, sin caer en el irracionalismo: no se trata de oponerse a la filosofía, sino de adecuar la filosofía a la realidad tal y como esta es. Y la verdad es antes un evento histórico-entitativo ( $\mathrm{y}$, por lo tanto, creatural-cristiano) que una construcción meramente abstractiva-racional. El culmen de este evento es el Crucificado (síntesis y centro - como se vio en la lectura de Von Balthasar- del espacio y de la historia); que se manifiesta como expresión culminante del amor del Padre creador, como redentor y como anuncio del Paráclito santificador; que se aparece a san Francisco, marcando un antes y un después en el íter de la historia cristiana; y que apunta hacia un final reductivo de los tiempos (la recapitulación de todas las cosas en Cristo). Por lo tanto, queda perfectamente trazado el marco suficiente para desarrollar una "teología de la historia". Esta teología de la historia, que para Ratzinger es la perspectiva más genuina del pensamiento bonaventuriano, supera también la tentación del pseudo-joaquinismo herético y dota de un nuevo sentido a la estructura teológica clásica de "los seis días" (Hexaëmeron), incorporando el "séptimo día" (reposo en Dios) de Joaquín de Fiore. Para Pandolfi, esta mirada del teólogo alemán es perfectamente compatible y continuadora de la lectura de Von Balthasar: tomando como núcleo histórico-salvífico la crucifixión del Verbum -expresión acabada del amor del Padre y causa de la salvación y de la santificación por el Espíritu-, la Trinidad aparece como modelo de toda la realidad en clave histórica (cuasi proódica, pero con un claro sentido anagogógico y escatológico): sea de todo el cosmos, sea de cada espíritu cristiano que vive y camina hacia el encuentro con $\operatorname{Dios}^{52}$.

3. "LA LECTURA BONAVENTURIANA DE ARISTÓTELES DESDE SAN FRANCISCO" 53 , MANUEL LÁZARO PULIDO.

El profesor especialista en el pensamiento de San Buenaventura y en la heredad teológica y filosófica franciscana, presenta un problema de partida: sus estudios se encuentran disgregados en un gran número de artículos, capítulos de libro y obras monográficas, cuyo análisis sistemático supera las restricciones del método con el que se aborda el presente trabajo. Por ello, el punto central del análisis se articulará en torno a varias de esas obras, aunque la tesis central aparezca con nitidez en un artículo publicado por el autor que se refiere

53 Lázaro Pulido, "La lectura bonaventuriana de Aristóteles desde san Francisco". 
explícitamente al tema que nos ocupa, en la revista "Pensamiento: Revista de investigación e Información filosófica” en el 2017.

La tesis de este artículo gira en torno a la problemática recepción de la obra del Estagirita en la Edad Media, que al comenzar a generar un pensamiento fértil (lleno de debates, comentarios y matices) produjo distintas "modulaciones" en su heredad intelectual. Un ejemplo claro de ello, para el autor del artículo, es precisamente san Buenaventura. Para Manuel Lázaro el debate sobre el "aristotelismo" o "antiaristotelismo" del Seráfico no parece poder concluirse si el problema se plantea de manera unilateral (como si se tratara de una mera relación entre dos autores). En este sentido Von Balthasar, en los textos ya citados en el punto precedente, parece dar con una clave de lectura adecuada ${ }^{54}$ : Buenaventura puede considerarse aristotélico si su aristotelismo se encuentra "modulado" por su franciscanismo. Concluye el autor:

"San Francisco es un paradigma, -el Modelo ${ }^{55}$ - que modula simbólicamente toda la realidad, enriquece el pensamiento aristotélico en manos de autores como san Buenaventura, profundizando los límites de un conocimiento tan claro como el de Aristóteles, desde la concepción de la sabiduría como meta de toda búsqueda de la verdad; en fin, un ejemplo de coexistencia y cooperación de universos diferentes en aras de la comprensión y construcción del mundo medieval" $" 56$.

A partir de esta interpretación base, en que el pensamiento del Estagirita fertiliza el de Buenaventura como un componente más en una tierra que es principalmente franciscana, es preciso limitar, también, la influencia del abad calabrés Joaquín de Fiore. En este sentido Manuel Lázaro critica la postura de Ratzinger. No se trata de una toma de postura contraria sino de un gesto de genuino acercamiento hermenéutico también al contexto del teólogo alemán. En efecto, en el preconcilio resultaba de particular relevancia el tratamiento "horizontal" del horizonte histórico-teológico en clave escatológica. Esta "insistencia eclesiológica de la teología" y la consiguiente "implicación histórica" explican el interés que suscita una lectura que es eminentemente escatológica, como la de Joaquín de Fiore. "Siendo la escatología en san Buenaventura un elemento de especial relevancia, la figura de Joaquín de Fiore tuvo que ejercer su

54 Cf. Lázaro Pulido, "La lectura bonaventuriana de Aristóteles desde san Francisco", 108.

55 Cf. Daniel Randolph, "Symbol or Model? St. Bonaventure's Use of St. Francis", Bonaventuriana: Miscellanea in Onore Di Jacques Guy Bougerol OFM, vol. I (Roma: Edizioni Antonianum, 1988), 55-62.

56 Lázaro Pulido, "La lectura bonaventuriana de Aristóteles desde san Francisco", 113-114. 
influencia" ${ }^{\text {"57 }}$. Sin embargo, para Manuel Lázaro es claro que Ratzinger yerra al concederle a este elemento una importancia capital. Especialmente en cuanto esta interpretación le lleva a situar sobre los hombros de Buenaventura una cierta carga antifilosófica. Para Manuel Lázaro es claro que no puede afirmarse ni que la teología de la historia sea el eje explicativo o estructural del pensamiento del Seráfico, ni que este pensamiento caiga, precisamente por ello, en formas de anti-filosofismo. El profesor extremeño, si bien nacido en Barcelona, se apoya como contrapeso en la tradición española reciente de estudiosos bonaventurianos, especialmente en los profesores Enrique Rivera de Ventosa y Bernardino de Armellada. Especialmente para el primero la propuesta de Ratzinger supone olvidar lo central del espíritu del Seráfico, y hurtar de su potencialidad la inteligencia mística de San Buenaventura.

"Y es ese olvido el que hace que la escatología tome un papel determinante, donde el alma individual es relegada en esta interpretación por el colectivo humano, traicionando, a su entender, el auténtico enfoque que se deja traslucir en los textos como el Itinerarium mentis in Deum. Este olvido del individual por el colectivo supone, además, un primer paso en la omisión del acontecimiento central de la Encarnación como lugar visible y central de la historia y de toda economía de salvación. El pensamiento escatológico en san Buenaventura es secundario frente a la realidad ya realizada en la encarnación de Cristo y en el acontecimiento extraordinario de revitalización que supone san Francisco" 58 .

En el mismo capítulo Manuel Lázaro reafirma el acierto de Von Balthasar a la hora de matizar la influencia joaquinista en el pensamiento bonaventuriano, al hacerla pasar por el tamiz del pensamiento franciscano -en la referencia ya citada-. De nuevo, para el estudioso extremeño, la influencia capital o la herencia principal en el pensamiento del Seráfico es la del Poverello. Sobre esta base, concluye, pueden incorporarse de forma adecuada y ordenada todos los demás elementos "heredados" -aristotélicos o joaquinistas- sin necesidad de acusar en su pensamiento formas de anti-filosofismo o de anti-teologismo.

La obra donde Manuel Lázaro analiza las posturas de Gilson y de Van Steenberghen es, principalmente, la monografía "La creación en san Buenaventura", especialmente en su interesante lectura sobre la -así llamada- "la

57 Manuel Lázaro Pulido, "La raíz joaquinista en san Buenaventura: lectura franciscana del pensamiento figurativo Joaquin de Fiore", Pensare per figure. Diagramme e simboli in Gioacchino da Fiore, Opere di Gioacchino da Fiore. Testi e strumenti 3 (Roma: Viella, 2010), 314.

58 Lázaro Pulido, "La raíz joaquinista en san Buenaventura", 315. La cursiva es del autor. En este punto Manuel Lázaro está glosando la reseña del profesor Rivera de Ventosa: Cf. Enrique Rivera de Ventosa, "Bibliografía”, Naturaleza y Gracia 8 (1961): 179. 
cuestión bonaventuriana": que supuso en el pensamiento del Seráfico la crisis del desarrollo del aristotelismo en las universidades del s.XIII y la consiguiente disputa entre quienes defendían la autonomía de la razón y quienes defendían su necesaria dependencia respecto a la fe. Cabe resaltar que, de los tres autores mencionados, el profesor cacereño, parece el único que aborda el contexto histórico desde una perspectiva global franciscana: señalando con claridad los distintos hitos históricos que supuso el desarrollo de la orden en relación con el progreso académico de las universidades en el siglo XIII ${ }^{59}$. Como señala, el punto de partida del debate lo establece Pierre Mandonnet, al situar el agustinismo como una corriente teológico-especulativa alternativa al aristotelismo "dominicano" de Tomás de Aquino y Alberto Magno. En esta gran familia se engloban tanto algunos dominicos (Roland de Cremona o Roberto Kilwardby), y la gran mayoría de franciscanos (Alejandro de Hales, Buenaventura, Jean de la Rochelle, etc.) y de seculares (Enrique de Gante, Guillermo de Alvernia, etc.). A pesar de presentar escuelas y formas de pensamiento bastante diversas entre sí, para Mandonnet todos ellos defienden una serie de puntos en común que los hace "agustinianos": ausencia de una distinción formal entre filosofia y teología $^{60}$, el punto de partida y la referencia filosófica fundamental es Platón -no Aristóteles- ${ }^{61}$, la preeminencia de la noción de "bien" sobre la de "verdad" ${ }^{62}$ y la actualidad positiva de la materia prima (no es pura potencialidad) ${ }^{63}$. Sin profundizar mucho más en su análisis, el dominico beumontés da por finalizada el muestrario de las corrientes de la filosofía medieval. Manuel señala cómo Gilson responde a este análisis algo simplista declarando al Seráfico como un "filósofo contrario al Estagirita". En efecto, para Gilson es posible que se dé este punto intermedio en la propuesta del agustinismo o de una "filosofía cristiana" de base franciscana. Para el filósofo e historiador parisino, la obra de Buenaventura -especialmente su metafísica- es "antiaristotélica" no tanto por un desconocimiento de la obra del Filósofo, cuanto por una opción preferencial por parte de san Buenaventura. En cierto modo sobre estos dos principios relacionados, pero no necesariamente- del "antiaristotelismo" y de la opción por una propuesta filosófica y metafísica compatible con la mística franciscana, el Seráfico lleva a cabo sea su proyecto filosófico, sea su síntesis teológica en las distintas obras a lo largo de su vida. La separación respecto al proyecto tomista

59 Cf. Manuel Lázaro Pulido, La creación en Buenaventura: acercamiento filosófico a la metafisica expresiva del ser finito, Pensadores cristianos (Grottaferrata: Frati editori di Quaracchi, 2005), 35-45.

60 Cf. Pierre Mandonnet, Siger de Brabant et l'averrö̈sme latin au XIIIme siècle; étude critique et documents inédits, par Pierre Mandonnet, O.P (Friburgo: Librairie de l'Université, 1899), 54-55.

61 Cf. Mandonnet, Siger de Brabant et l'averroïsme latin, 55-56.

62 Cf. Mandonnet, Siger de Brabant et l'averroüsme latin, 56.

63 Cf. Mandonnet, Siger de Brabant et l'averroïsme latin, 56-57. 
permanece, pero no como un elemento que desdice del Seráfico, sino como un dato que lo distingue y lo convierte "(...) en el juez privilegiado del Filósofo" ${ }^{64}$. Para Manuel la propuesta de Gilson es aceptable en sentido amplio, pero no en sentido estricto. Por ejemplo, la expresión "filosofía franciscana" es aceptable como una propuesta de vida derivada de la "forma mentis" que caracteriza a la Orden. Otro problema que surge de la interpretación del filósofo parisino es la forzada contraposición con el aristotelismo (contraposición extremada por $\mathrm{J}$. Ratzinger -remacha el autor-). Para Manuel Lázaro este pretendido "antiaristotelismo" es muy difícil de demostrar e incluso parece contradecir numerosos pasajes y afirmaciones del Seráfico.

Van Steenberghen responde a Gilson "(...) dando un giro significativo a la filosofía bonaventuriana"65. Para Manuel Lázaro el punto central de desacuerdo estriba, precisamente en las expresiones "metafísica de la mística" o "filosofía franciscana", que para el belga no solo no son extrañas al pensamiento de buenaventura, sino que carecen incluso de sentido. Estos conceptos denotan una postura hostil por parte de Buenaventura hacia el aristotelismo. La postura de Gilson y de Mandonnet suponen la existencia de una corriente filosófica cátalogable como "agustinismo" o "filo-platonismo". Tales corrientes, defiende Van Steenberghen, son inexistentes. Lo que sí puede encontrarse en las universidades del siglo XIII es una corriente creciente de aristotelismo que se divulga e interpreta. El historiador y filósofo lovaniense acepta que la influencia del aristotelismo en la teología bonaventuriana no es tan relevante como la influencia del franciscanismo o del agustinismo, mientras que para la filosofía resulta indispensable como "instrumento racional de la ciencia sagrada: "La labor filosófica se integra en la unidad del saber cristiano como "etapa de adquisición de la sabiduría integral" 66 . Por tanto, para Van Steenberghen, Buenaventura es una suerte de aristotélico neoplatónico bastante ecléctico. Para Manuel Lázaro esta interpretación peca gravemente al suponer una deuda insuperable respecto a la influencia aristotélica predominante de Tomás de Aquino. De hecho, en cierto modo, parece que la forma más correcta de interpretar adecuadamente el pensamiento del Seráfico en la "cuestión bonaventuriana" consistiría en una suerte de síntesis crítica de estas dos últimas interpretaciones, incluyendo el telón de fondo que señalara Von Balthasar ${ }^{67}$.

64 Lázaro Pulido, La creación en Buenaventura, 47. La cursiva es del autor.

65 Lázaro Pulido, La creación en Buenaventura, 47.

66 Lázaro Pulido, La creación en Buenaventura, 48. El autor hace referencia a Fernand Van Steenberghen, Filosofia medieval (Buenos Aires: Club de Lectores, 1968).

67 Cf. Lázaro Pulido, La creación en Buenaventura, 49-51. El autor destaca como otras interpretaciones posteriores y más adecuadas las de T. de Andrés, P. Robert y A. Poppi. 


\section{DISCUSIÓN}

Dicho lo cual, las conclusiones que se pueden sacar del análisis de Speer sobre los principales intelectuales del siglo XX que han estudiado la relación entre el pensamiento de Buenaventura entre filosofía y teología (y, por ende, de su recepción de Aristóteles):

1. Los historiadores de la filosofía suelen errar al enfatizar el proceso de independencia de la filosofía respecto a la teología durante los siglos XIII y XIV como si se tratara de un proyecto programado. Bajo esta perspectiva otorgan una importancia radical a las condenas de 1270 y de 1277. A la luz de estos acontecimientos el Seráfico aparece casi como un ideólogo revolucionario antiaristotélico. Esta visión no concuerda con una lectura sistemática de sus obras de madurez.

2. Gilson acierta al presentar la obra del Seráfico como una "síntesis medieval" (además de admitir su monografía sobre Buenaventura como la principal obra del género). Pero yerra al insistir en quitar al Seráfico la etiqueta de "filósofo" por no adaptar la estructura de su pensamiento al "Canon". En su afán por distinguir o separar el pensamiento de Buenaventura del de Tomás de Aquino, el filósofo e historiador parisino cae en una visión algo exagerada de la postura fundamental de ambos.

3. Van Steenberghen acierta en su crítica a Gilson y en su crítica a las interpretaciones que enfatizan la vocación franciscana del pensamiento de buenaventura. Sin embargo, el filósofo belga tampoco acierta a la hora de enfatizar el "aristotelismo bonaventuriano". Para Speer la síntesis medieval del Seráfico se nutre casi a la par de muchas fuentes diversas, pero el modus parece ser más agustiniano en el enfoque.

4. Por último, en las pocas menciones que se hacen a la propuesta de Ratzinger, el autor ve sus aportaciones con un severo espíritu crítico, especialmente por la exagerada "teoría evolutiva" del pensamiento bonaventuriano y por el énfasis en la óptica anti-filosófica y anti-aristotélica al analizar la obra de madurez del Seráfico. Aunque Ratzinger acierta al proponer la interpretación de las Collationes in Hexaëmeron como una síntesis teológica desde la perspectiva de la historia de la salvación.

Del análisis de Carmelo Pandolfi se pueden sacar las siguientes conclusiones:

1. Para É. Gilson la fuente del pensamiento bonaventuriano es San Agustín. La originalidad del Seráfico consiste en descubrir que dentro del "Evento-Cristo" 
se re-entrega al pensamiento la posibilidad de una "reductio" resolutiva hacia Dios (Principio-Fin). Para ello, según Gilson, Buenaventura debe contradecir al "Aristóteles de moda" en todo aquello cuanto niega la posibilidad de este retorno "in Deum" o todo cuanto afirma la autonomía radical de la filosofía respecto a la teología. En este sentido el tomismo y el agustinismo bonaventuriano son los únicos caminos contra las tentaciones de este aristotelismo latino. La crítica de Pandolfi a esta propuesta es doble: Gilson no es del todo justo con el patrimonio aristotélico de Buenaventura y no ve en Buenaventura una verdadera "superación" del pensamiento agustiniano.

2. Para Van Steenberghen, la referencia clara del pensamiento de Buenaventura es Tomás de Aquino: ambos comparten fundamentalmente el mismo proyecto y el Aquinate descuella por su lucidez. En San Buenaventura, como "Magister Sacra Doctrina" no se da una verdadera independencia de la "ratio". A pesar de lo cual el Seráfico logra una síntesis de aristotelismo (sínolo) y de neoplatonismo (participación), a la tomasiana. De hecho, ambos medievales son caras de la misma moneda: el aristotelismo latino. Para Pandolfi, el belga yerra al exagerar la distancia entre Agustín y Buenaventura y también se equivoca al negar en san Buenaventura una propuesta de síntesis de filosofía cristiana propia.

3. La gran aportación de Von Balthasar consiste en una nueva clave hermenéutica con dos polos la belleza trascendental y la teología de la Trinidad. El punto central es Cristo como expresión acabada del amor del Padre. La metafísica del Bien en Buenaventura es fácilmente compaginable con lo mejor del tomismo si se ve la analogía del ente creatural dentro del contexto trinitario del amor absoluto. Así, el teólogo de Lucerna, vincula a Buenaventura más con Pseudo Dionisio Areopatiga y con san Francisco que con san Agustín o Aristóteles.

4. Para Pandolfi en la visión de Ratzinger se descubre un "pseudo-antiaristotelismo", que es realmente un "anti-filosofismo". San Buenaventura tiene más interés en el carisma franciscano que en el método o el problema escolástico. Por eso su cénit se encuentra en la "teología de la historia". Este es el marco teórico adecuado para interpretar su propuesta teológica y filosófica (el "horizonte ontológico integral" de su pensamiento). Para Ratzinger, las principales fuentes de Buenaventura son San Francisco, Joaquín de Fiore y san Agustín. La distancia respecto a Tomás de Aquino o Aristóteles es muy amplia, prácticamente de base. 
Del análisis de Manuel Lázaro Pulido pueden sacarse las siguientes conclusiones:

1. El telón de fondo para resolver adecuadamente la "cuestión bonaventuriana" requiere tener en cuenta tanto la tradición carismática franciscana que da sentido a su proyecto filosófico y teológico, como la influencia del despertar y del desarrollo del pensamiento aristotélico en un contexto teológicointelectual (el universitario del s. XIII) dominado por el pensamiento agustiniano.

2. H.U. Von Balthasar tiene el gran mérito de haber sabido dibujar escueta, pero bellamente el horizonte amplio de desarrollo del pensamiento del Seráfico, señalando un orden prioritario de influencia que parece, al menos por lo general, adecuado.

3. En cambio J. Ratzinger se equivoca por partida doble, sea al afirmar tajantemente el "antiarisotelismo" y "antifilosofismo" en el pensamiento de san Buenaventura, sea en otorgar excesiva importancia (claramente marcado por sus circunstancias vitales y biográficas) a la teología de la historia de Joaquín de Fiore. En ningún caso puede considerarse el pensamiento bonaventuriano como estructuralmente centrado en una forma de "teología de la historia".

4. É. Gilson acierta al corregir el desdén de P. Mandonnet acerca de la "filosofía agustiniana" y sitúa el pensamiento bonaventuriano entre una especie de "antiaristotelismo", que no supone un rechazo de toda forma de filosofía, y un pensamiento definido como "metafísica de la mística" o "filosofía franciscana" (que, en rigor, a juicio de Manuel Lázaro, no parecen formas de filosofía). El enfrentamiento de Buenaventura y Aristóteles parece, por su propia cuenta, un punto muy problemático y muy difícil de defender.

5. Para Manuel Lázaro parte de este defecto lo corrige F. Van Steenberghen, que se opone en ambos puntos a lo propuesto por Gilson. Para el filósofo e historiador belga Buenaventura es una "especie de aristotélico neoplatónico bastante ecléctico". Sin embargo, Manuel Lázaro juzga excesiva la importancia con la que Van Steenberghen valora la influencia de Tomás de Aquino en el pensamiento del Seráfico. Ante la propuesta unificadora de una filosofía y una teología compactas en el pensamiento bonaventuriano (hipótesis de Gilson), Van Steenberghen reivindica el lema "philosophia ancilla theologiae". Postura esta última en el debate sobre la cuestión bonaventuriana que supone una cierta exageración de la relación entre aristotelismo y agustinismo en el pensamiento del Seráfico. 
Dicho lo cual resulta fácil presentar algunas conclusiones:

1. La dificultad del problema de la recepción de Aristóteles por san Buenaventura (que abre la puerta al problema de la relación entre fe y razón), no ha quedado del todo zanjada. Entre los estudiosos de hoy en día existe un cierto consenso en algunos puntos (las posturas de Gilson, Ratzinger y Van Steenberghen han quedado algo superadas), aunque también existen puntos de desacuerdo (la influencia del franciscanismo, del joaquinismo, del agustinismo o cuál es la influencia predominante en el pensamiento del Seráfico a la hora de "matizar" la novedad del aristotelismo y cuáles son las aportaciones originales del mismo Buenaventura).

2. Sin el mismo rigor de juicio los tres estudiosos analizados - de tres contextos universitarios diversos- parecen considerar como al menos parcialmente superados los estudios de É. Gilson, de F. Van Steenberghen y de J. Ratzinger. Para Pandolfi, el primero y el tercero más que superados requieren un cierto "aggiornamento" al debate actual. En cambio, Speer y Lázaro parecen acercarse más a la postura de Van Steenberghen, matizando debidamente sus exageraciones sobre la influencia de Tomás de Aquino en el pensamiento de San Buenaventura. Estas distintas apreciaciones se deben tanto a la perspectiva particular de cada estudioso, como al marco más amplio del trabajo en el que se realiza tal análisis.

3. Speer enmarca su análisis general en una afirmación amplia: el análisis histórico o filosófico de la "cuestión bonaventuriana" no debe obviar que la autonomía de la razón no es un pecado medieval, sino moderno. Es decir, que la cuestión de la relación entre "verdad racional" y "verdad de fe" no debe enfocarse desde la perspectiva del problema moderno, sino desde la mirada sintética que caracteriza la escolástica medieval. Pandolfi en cambio formula su taxonomía de interpretaciones bonaventurianas dentro de una investigación más amplia sobre los elementos fundamentales que caracterizan las distintas formas de pensamiento cristiano. Por ello, encuentra particular riqueza en la consideración de la belleza como síntesis del "Itinerarium" bonaventuriano que presenta Von Balthasar. El caso de Manuel Lázaro es problemático, debido a la cantidad de fuentes a las que es preciso recurrir para formular un juicio suficiente. Parece que la síntesis o el punto de referencia de su perspectiva, se encuentra en el artículo "La lectura bonaventuriana de Aristóteles desde san Francisco". Otro gran valor del profesor extremeño estriba en la gran cantidad de fuentes secundarias referenciadas.

4. Por lo tanto se puede constatar, como valor conclusivo, una tradición viva que se construye y se desarrolla, que comienza a formar bases interpretativas aceptadas por el consenso general, pero que a la vez somete a juicio crítico el 
valor de las distintas opiniones (voces tan autorizadas con Gilson o Ratzinger), sin caer nunca en una de las grandes catástrofes de la herencia tomista: el abandono de los textos originales del Angélico. En efecto, se constata en todos los estudiosos mencionados o referenciados (a excepción de P. Mandonnet) un gran dominio de toda o gran parte de la bibliografía del Seráfico.

5. Parece posible plantearse, a partir de esta tradición de pensamiento, sea una mayor especialización en problemas concretos dentro de la hermenéutica del pensamiento bonaventuriano (por ejemplo, la olvidada heredad de Alejandro de Hales, denunciada por Speer), sea una continuación o desarrollo del pensamiento bonaventuriano en diálogo o debate con los problemas filosóficos y teológicos de la actualidad.

\section{REFERENCIAS BIBLIOGRÁFICAS}

Aertsen, Jan A. "Gibt Es Eine Mittelalterliche Philosophie". Philosophisches Jahrbuch 102 (1995): 161-176.

Agustín de Hipona. Sermones XLIII. PL 38.

Aristóteles. Metafisica. Editado por W.D. Ross. Oxford: Clarendon Press, 1924. Aubenque, Pierre. "Aristóteles y el problema de la metafísica". Logos. Anales del Seminario de Metafisica 50 (2017): 9-19.

Buenaventura. III Sententiarum, Ed. Quaracchi, III.

Buenaventura, Collationes de septem donis Spiritus. Ed. Quaracchi, V.

Buenaventura, Collationes in Hexaëmeron. Ed. Quaracchi, V.

Buenaventura, Sermones selecti de rebus theologici, sermo IV. Ed. Quaracchi, $\mathrm{V}$.

De Libera, Alain. La philosophie médiévale. Paris: Presses universitaires de France, 1993.

De Libera, Alain. Penser au Moyen Age. Paris: Editions du Seuil, 1991.

Flasch, Kurt y Tempier, Etienne. Aufklärung im Mittelalter?: Die Verurteilung von 1277: das Dokument des Bischofs von Paris. Mainz: Dieterich, 1989.

Gilson, Étienne. La Filosofia de San Buenaventura. Buenos Aires: Desclée de Brouwer, 1948.

Gilson, Étienne. La filosofía en la Edad Media. Madrid: Editorial Gredos, 2014. Imbach, Ruedi. Gli Studi Di Filosofia Medievale Fra Otto E Novecento. Roma: Ed. di Storia e Letteratura, 1991.

Juan de Salisbury. Metalogicum, III. PL 199. 
Lázaro Pulido, Manuel, et al. Historia de la filosofía medieval y renacentista I. Madrid: Editorial UNED-Sindéresis, 2018.

Lázaro Pulido, Manuel. La creación en Buenaventura: acercamiento filosófico a la metafísica expresiva del ser finito. Grottaferrata: Frati editori di Quaracchi, 2005.

Lázaro Pulido, Manuel. "La lectura bonaventuriana de Aristóteles desde san Francisco". Pensamiento: Revista de investigación e Información filosófica 275 (2017): 103-114.

Lázaro Pulido, Manuel. "La raíz joaquinista en san Buenaventura: lectura franciscana del pensamiento figurativo Joaquin de Fiore". Pensare per figure. Diagramme e simboli in Gioacchino da Fiore, 314-340. Roma: Viella, 2010).

León Florido, Francisco. Las filosofias en la Edad Media: crisis, controversias y condenas. Madrid: Biblioteca Nueva, 2010.

Mandonnet, Pierre. Siger de Brabant et l'averrö̈sme latin au XIIIme siècle; étude critique et documents inédits, par Pierre Mandonnet, O.P. Friburgo: Librairie de l'Université, 1899.

Pandolfi, Carmelo. Forme del pensare cristiano: Agostino, Anselmo, Bonaventura, Tommaso. Roma: If Press, 2016.

Randolph, Daniel. "Symbol or Model? St. Bonaventure's Use of St. Francis", Bonaventuriana: Miscellanea in Onore Di Jacques Guy Bougerol OFM, vol. I. Editado por Francisco de A. Chavero, 55-62. Roma: Edizioni Antonianum, 1988 ,

Ratzinger, Joseph. Obras completas de Joseph Ratzinger, II: Comprensión de la revelación y teología de la historia de San Buenaventura, vol. II. Madrid: Biblioteca de Autores Cristianos, 2013.

Rivera de Ventosa, Enrique. "Bibliografía". Naturaleza y Gracia 8 (1961).

Speer, Andreas. "Bonaventure and the Question of a Medieval Philosophy". Medieval Philosophy \& Theology 6 (1997): 25-46.

Van Steenberghen, Fernand. Aristotle in the West: the origins of latin Aristotelianism $=$ Aristote en Occident. Louvain: Nauwelaerts Publishing House, 1970.

Van Steenberghen, Fernand. Epistémologie. Louvain: Ed. de l'Inst. Supérieur de Philosophie, 1956.

Van Steenberghen, Fernand. Filosofía medieval. Buenos Aires: Club de Lectores, 1968. 
Van Steenberghen, Fernand. La philosophie au XIIIe siècle. Louvain-la-Neuve: Institut Superieur de Philosophie, 1991.

Von Balthasar, Hans Urs. Gloria. Una estética teológica, Estilos eclesiásticos. Ireneo, Agustín, Dionisio, Anselmo, Buenaventura, vol. II. Madrid: Encuentro, 1992.

Francisco Javier Rubio Hípola

Departamento de Humanidades Universidad Francisco de Vitoria Carretera Pozuelo a Majadahonda, Km 1.800, 28223 Pozuelo de Alarcón, Madrid (España) https://orcid.org/0000-0003-0859-7852 